\title{
Contact Injection into Polymer Light-Emitting Diodes
}

\author{
E. M. Conwell and M. W. Wu \\ Center for Photoinduced Charge Transfer, Chemistry Department, University of Rochester, \\ Rochester, New York 14627 \\ and Xerox Corporation, Wilson Center for Technology, 114-22D, Webster, New York 14580
}

\begin{abstract}
The variation of current $I$ with voltage $V$ for poly(phenylene vinylene) and other polymer light-emitting diodes has been attributed to carriers tunneling into broad conduction and valence bands. In actuality the electrons and holes tunnel into polaron levels and transport is by hopping among these levels. We show that for small injection the $I-V$ characteristic is determined mainly by the image force, for large injection by space charge effects, but in both cases the strong variation of mobility with field due to disorder plays an important role.
\end{abstract}

PACS: $73.40 . \mathrm{Ns}, 72.20 . \mathrm{Ht}, 71.20 . \mathrm{Hk}$ 
To improve the efficiency of polymer light-emitting diodes, LEDs, it is essential to understand and improve performance of the contacts. Contact injection into LEDs made of MEH-PPV [poly(2-methoxy,5-(2'-ethyl-hexoxy)-1,4-phenylene-vinylene] has been attributed to tunneling of the electrons and holes into a broad conduction or valence band, respectively, in the polymer through interface barriers arising from the band offset between the polymer and the metal electrodes. ${ }^{1,2}$ Good agreement of the $I-V$ characteristic with the field dependence given by Fowler-Nordheim tunneling has been shown in references 1 and 2, although others did not find the Fowler-Nordheim field dependence for their diodes.

The picture of carriers tunneling into a wide band cannot be correct for the conducting polymer samples now available. First, because of the short conjugation lengths for typical polymers such as PPV, on average $\sim 6$ or 7 monomers, continuum-like bands become sets of discrete levels. For the average conjugation length the level spacing is $\sim$ several $k T$ at room temperature. ${ }^{3,4}$ A more serious objection, however, is that injection of electrons or holes is into polaron levels, ${ }^{5,6}$ which for electrons lie below the LUMO and for holes above the HOMO. The separation of a polaron level from the LUMO or HOMO depends on the conjugation length of the segment. For PPV calculations give the separation as $0.15 \mathrm{eV}^{7}$ or $0.2 \mathrm{eV}^{3}$ for very long segments, increasing to $\sim 0.7 \mathrm{eV}$ for a 3 monomer long segment. It is also a consequence of the short conjugation lengths that theories based on the formation of a bipolaron lattice in the neighborhood of the contact cannot apply to currently available conducting polymer samples. 8

Based on the above considerations the scenario for conduction in an LED begins with a carrier from the metal tunneling into a polaron level close to the contact. The carrier then diffuses and hops in the field from one conjugation length to another. The variation in conjugation lengths, and the presence of defects, result in a spread in energy of the hopping sites (diagonal disorder). An appropriate model for treating the transport in this system for low injection is the disorder model pioneered by Bässler and associates. 9 The distribution in energy of the polaron states is taken as a Gaussian with variance $\sigma$. 0 From the expected spread in conjugation lengths it is reasonable that $\sigma \sim 0.1 \mathrm{eV}$. 
Critical for the behavior of a contact is the location in energy of the polaron states of the polymer relative to the Fermi energy $E_{F}$ of the metal. For specificity we will discuss the case of electron injection into the polymer but the results apply to hole injection with the usual modifications. Internal photoemission measurements, such as those of Campbell et $a l,{ }^{5}$ yielding the energy required to inject an electron from a metal into a polymer, give the energy difference between $E_{F}$ and some average state in the polaron distribution. We denote this energy by $W$. In what follows we calculate $I-V$ characteristics for a case of large $W$, which means small injection, using the results of a Monte Carlo simulation based on the disorder model. 11 We then carry out a calculation for $W \simeq 0$, which is the case for calcium contacts on MEH-PPV, ${ }^{5}$ using the classical approach of Rose ${ }^{22}$ and Lampert. 13 In the latter case agreement is obtained, for reasonable values of the parameters involved, with the $I-V$ characteristic of samples with only a Ca contact injecting, providing we take into account the strong variation of mobility with field documented for PPV by Karg et al.

In the Monte Carlo simulation 11 the energy $U$ of a polaron site as a function of the perpendicular distance $x$ from the metal-polymer interface is written

$$
U(x)=W-e E x-e^{2} / 4 \kappa x
$$

Here $E$ is the electric field intensity and the last term represents the image force, $\kappa$ being the dielectric constant. In the presence of energetic disorder $U(x)$ gives the value of mean energy $\bar{\varepsilon}(x)$ of polaron sites at a distance $x$ from the interface. Note that all energies are measured relative to $E_{F}$. With $W$ large, e.g. $0.6 \mathrm{eV}$ or greater, injection is small and space charge may be neglected. To calculate the incoming flux of carriers we have assumed that they tunnel into a polaron level with energy $\varepsilon$ at a distance $x$ from the interface at the rate $v_{m}(x) \exp (-\varepsilon / k T)$, where $v_{m}(x)$ is a distance-dependent prefactor. With this assumption the total number of carriers tunneling into polaron levels per second is $v_{m}(x) \exp \left[\left(-\bar{\varepsilon}(x)+\sigma^{2} / 2 k T\right) / k T\right]$. Thus the energy distribution of initially populated sites is displaced by $\sigma^{2} / k T$ from the available site distribution. By Monte Carlo simulation we followed the hopping of the carriers through a sample of 12 layers, $331 \times 331$ sites/layer, 
finally obtaining the yield, i.e., the fraction of injected carriers that escape the return to the electrode and reach the opposite boundary of the sample.11 Even in the absence of disorder the image force results in the great majority of carriers returning to the electrode at low fields. An analytic solution for the ordered case based on Eq. (1) (neglecting space charge) 15.16 gives the yield as $0.3 \%$ in a field of $1.25 \times 10^{5} \mathrm{~V} / \mathrm{cm}$ at $300 \mathrm{~K}$ for the parameters of Ref. 11. A field $\sim 10$ times as large is required for essentially complete collection. The results of the analytic solution were in good agreement with the Monte Carlo simulation for $\sigma=0$, indicating that the simulation sample was thick enough. 1 In the disordered case the carriers have to overcome the random barriers due to disorder as well as the image force. The result is that at fields of $\sim 10^{5} \mathrm{~V} / \mathrm{cm}$ mild disorder $(\sigma=0.08 \mathrm{eV})$ results in a yield smaller by a couple of orders of magnitude even though the injection is larger. In addition the yield increases more strongly with increasing field. 1 Current vs. field may be obtained by multiplying the yield by the number of carriers entering the polymer per second. As shown in Fig. 1, for $W=0.7 \mathrm{eV}$ and a sample length of $120 \mathrm{~nm}$, the effects of the image force and moderate disorder are a current increase by a factor of $10^{5}$ as the applied voltage goes from 1 to 20 volts.

The photoemission data of Ref. 5 give the Fermi level for Ca lying above the polaron level in MEH-PPV by $0.05 \mathrm{eV}$. This suggests that Ca provides an ohmic contact on MEHPPV, meaning that it could supply the maximum required current, i.e., space-charge limited current. Calculations of current vs voltage for ohmic contacts were carried out in Refs. 12 and 13. The calculations are based on Poisson's equation:

$$
(\kappa / e)(d E / d x)=(n-\bar{n})+\left(n_{t}-\bar{n}_{t}\right),
$$

where $n$ and $n_{t}$ are the densities of free and trapped electrons, respectively, and $\bar{n}$ and $\bar{n}_{t}$ their respective average values for the sample in thermal and electrical equilibrium with the contact (no applied voltage). The equation for current density $J$ is simplified by neglecting the diffusion terms as was done in Ref. [13. In this case we include the field dependence of mobility $\mu$ found for hopping in many disordered systems, including holes in PPV, 14 to give 
for the steady current

$$
J=n e \mu_{0} e^{\alpha \sqrt{E}} E
$$

where $\mu_{0}$ is the zero-field mobility and $\alpha$ was taken as a parameter in the calculations. With the simplification of neglecting diffusion current the boundary condition at the cathode interface is $E=0$ at $x=0$. The other electrode is taken as non-injecting.

Although there is an estimate of the trap density in MEH-PPV, specifically a few times $10^{16} / \mathrm{cm}^{3}, 17$ we lack information about their location. We therefore carried out numerical integration of Eqs. (2) and (3) for trap-free $\left(n_{t}=\bar{n}_{t}=0\right)$ and all-traps-filled $\left(n_{t}=N_{t}\right.$, the total trap density ) cases. As discussed by Lampert, the trap-filled case does not give the correct current at low fields. Instead there is a voltage threshold for current flow because before voltage is applied there is already unneutralized charge in the traps which prevents the injection of additional charge at the electrodes. When $N_{t} \gg \bar{n}$, as is likely to be the case here, the current rises very steeply with voltage beyond the threshold. Nevertheless the trap-filled solution should be good at high enough fields.

In Fig. 2 we compare our calculated results with the experimental data of Parker for a Ca contact on MEH-PPV in the electrons-only case, i.e., with the work function of the other electrode too low to contribute significant hole current (at least below $18 \mathrm{~V})^{1}$. It is seen that good fits can be obtained for both the trap-free and trap-filled calculations, although, as anticipated, there is no fit at the lower fields in the latter case. For the trap-free case the parameters for the fit shown are $\alpha=8 \times 10^{-3} \mathrm{~cm}^{1 / 2} / \mathrm{V}^{1 / 2}$ and $\mu_{0}=5 \times 10^{-11} \mathrm{~cm}^{2} / \mathrm{Vs}$. For the trap-filled cases both solid and dashed lines correspond to $\mu_{0}=5 \times 10^{-9} \mathrm{~cm}^{2} / \mathrm{Vs}$. For the solid line the other parameters are $\alpha=4 \times 10^{-3} \mathrm{~cm}^{1 / 2} / \mathrm{V}^{1 / 2}$ and $N_{t}-\bar{n}_{t}-\bar{n}=6 \times 10^{16}$ $/ \mathrm{cm}^{3}$, while for the dashed line $\alpha=4.5 \times 10^{-3} \mathrm{~cm}^{1 / 2} / \mathrm{V}^{1 / 2}$ and $N_{t}-\bar{n}_{t}-\bar{n}=10^{17} / \mathrm{cm}^{3}$. Smaller $\mu_{0}$ is required for the fit to the trap free case because traps are not present to keep the current down at a given field. The parameter values are reasonable, particularly those for the solid line in the trap-filled case. Extrapolation of the $\mu$ vs $E$ data for holes of Ref. 14 to $E=0$ yields $\mu_{0}=5 \times 10^{-9} \mathrm{~cm}^{2} /$ Ss. Electron mobility is thought to be considerably 
lower than hole mobility in PPV, the difference most likely being due to deep traps (perhaps carbonyls) for the electrons. It is not unreasonable that with Ca contacts the deep traps are filled and electron mobility becomes comparable to hole mobility. Karg et al. obtain $\alpha=6 \times 10^{-3} \mathrm{~cm}^{1 / 2} / \mathrm{V}^{1 / 2}$, close to the $\alpha$ values obtained here. Finally, since $\bar{n}$ is expected to be small, $N_{t}-\bar{n}_{t}$ is close to the trap density estimated by Campbell et al from the magnitude of the initial increase in capacitance with forward bias. 17 From the good fit at high voltages we conclude that the current there is space-charge limited current. It does not vary as $\mathrm{V}^{2}$ because of the strong field dependence of $\mu$. We note that the importance of space charge effects has also been stressed, albeit within the Fowler-Nordheim model, by Davids et al. 8

In summary, we have pointed out that Fowler-Nordheim tunneling can not describe contact injection into currently available polymer samples because the short conjugation lengths mean they cannot have broad bands. Injection and transport involve only polaron levels. These have a spread in energy due to the conjugation length variations and other defects. This disorder, even though relatively mild, can decrease injection by a couple of orders of magnitude and makes mobility highly field dependent. Ca contacts to MEH-PPV should be ohmic because $E_{F}$ lies above the polaron level. $I-V$ characteristics resulting from injection at $\mathrm{Ca}$ contacts are well fitted by theory for space-charge limited current with reasonable values for the mobility and its variation with field, and the trap density.

We are grateful to Dr.Yu. Gartstein for valuable discussions and a critical reading of the manuscript. We acknowledge the support of the National Science Foundation under Science and Technology Center grant CHE912001. 


\section{REFERENCES}

${ }^{1}$ I.D. Parker, J. Appl. Phys. 75, 1656 (1994).

2 D. Braun and A.J. Heeger, Appl. Phys. Lett. 58, 1982 (1991).

${ }^{3}$ H.A. Mizes and E.M. Conwell, Synth. Met. 68, 145 (1995).

${ }^{4}$ S. Mazumdar and M. Chandross, Proc. SPIE 25-28, 62 (1995).

${ }^{5}$ I.H. Campbell, T.W. Hagler, D.L. Smith, and J.P. Ferraris, Phys. Rev. Lett. 76, 1900 (1996).

${ }^{6}$ A.J.Heeger, "Nature of the Primary Photoexcitations in Poly(arylene-vinylenes)" in "Primary Photoexcitations in Conjugated Polymers", N.S.Sariciftci, ed., World Scientific, preprint.

${ }^{7}$ J. Cornil, D. Beljonne and J.L. Bredas, J. Chem. Phys. 103. 834 (1995).

${ }^{8}$ P.S. Davids, A. Saxena, and D.L. Smith, Phys. Rev. B 53, 4823 (1996).

${ }^{9}$ H. Bässler, phys. stat. sol. (b) 175, 15 (1993).

${ }^{10}$ E.M. Conwell and Yu.N. Gartstein, SPIE 2528, 23 (1995).

${ }^{11}$ Yu.N. Gartstein and E.M. Conwell, Chem. Phys. Lett. 255, 93 (1996).

${ }^{12}$ A. Rose, Phys. Rev. 97, 1538 (1955).

${ }^{13}$ M.A. Lampert, Phys. Rev. 103, 1648 (1956).

${ }^{14}$ S. Karg, V. Dyakonov, M. Meier, W. Riess, and G. Paasch, Synth. Met. 67, 165 (1994).

${ }^{15}$ F. Willig, Chem. Phys. Lett. 40, 331 (1976).

${ }^{16}$ K.P. Charlé and F. Willig, Chem. Phys. Lett. 57, 253 (1978).

${ }^{17}$ I.H. Campbell, T.W. Hagler, D.L. Smith, and J.P. Ferraris, Phys. Rev. Lett. 76, 1900 (1996). 
18 P.S. Davids, Sh.M. Kogan, I.D. Parker, and D.L. Smith, Appl. Phys. Lett. 69, 2270 (1996). 


\section{FIGURES}

FIG. 1. Current density vs voltage from Monte Carlo simulation for a disordered sample $(\sigma=0.08 \mathrm{eV})$ with one injecting contact with large $W(0.7 \mathrm{eV})$. Sample length is $120 \mathrm{~nm}$.

FIG. 2. The dots represent $I$ - $V$ data from Ref. 1 for $120 \mathrm{~nm}$ long MEH-PPV LEDs with one Ca contact and the other contact $\mathrm{Mg}(\bullet)$ or $\mathrm{Nd}(\mathrm{o})$. The lines represent theoretical fits to the data for the trap-free and trap-filled cases. 